\title{
An Analysis of Registration of Brain Images using Fast Walsh Hadamard Transform
}

\author{
D.Sasikala \\ Assistant Professor \\ Department of Computer Science and Engineering \\ Bannari Amman Institute of Technology \\ Sathyamangalam, Tamil Nadu-638401.
}

\author{
R.Neelaveni \\ Associate Professor \\ Department of Electrical and Electronics Engineering \\ PSG College of Technology \\ Coimbatore, Tamil Nadu -641004.
}

\begin{abstract}
A bundle of image registration procedures have been built up with enormous implication for data analysis in medicine, astrophotography, satellite imaging and little other areas. This problem proposes a solution using a technique for medical image registration using Fast Walsh Hadamard transform. This algorithm registers the images of the mono or multi modalities. Each image bit is expanded in terms of Fast Walsh Hadamard basis functions. Each basis function is an idea of resolving a choice of features of local structure, e.g., horizontal edge, corner, etc. These coefficients are normalized and used as digits in a preferred number system which lets one to outline a unique number for all type of local structure. The research outcomes confirm that Fast Walsh Hadamard transform realized better results than the traditional Walsh transform in the time domain. Also Fast Walsh Hadamard transform is further reliable in medical image registration devastating less time.
\end{abstract}

\section{General Terms}

Image Processing, Brain Images, Image Registration, Medical Image Registration.

\section{Keywords}

Walsh Transform, Fast Walsh Hadamard Transform, Local Structure, Normalization, Mutual Information, Correlation Coefficient.

\section{INTRODUCTION}

Digital image processing is developing the eventual machine that could carry out the visual functions of all. It is a swiftly budding field with developing applications in many areas of Science and Engineering. The most important principle of registration is to combine the sets of data with the variants if any or with their similarities into a single data. These sets of data are got by sampling the identical scene or object at different times or from different perspectives, in different co-ordinate systems. The intention of registration is to envision a single data merged with all the details about these sets of data obtained at different times or perspectives or co-ordinate systems. Such data is very crucial in medicine for doctors to prepare for surgery. The most general and significant classes of image analysis algorithm with medical applications [1,3] are image registration and image segmentation. In image analysis procedure, the same input gives out somewhat detail sketch of the scene whose image is being considered. Hence the image analysis algorithms execute registration as a part of it headed for generating the depiction. In solitary subject analysis, the statistical analysis is made either prior to or subsequent to registration. But in group analysis, it is completed subsequent to registration.

Commonly registration is the most complex task, as aligning images to have common characteristics by general features and differences if any are to be stressed for immediate visibility to the naked eye. There is no universal registration [1-18] algorithm that can work reasonably well for all images. An appropriate registration algorithm for the particular problem must be chosen or developed, as they are adhoc in nature. The algorithms can be integrated explicitly or implicitly or even in the form of a various of factors. This step establishes success or failure of image analysis. This procedure may be classified based on four different aspects given as follows: (i) the feature selection (extracting features from an image) using their similarity measures and a correspondence basis (ii) the transformation function (iii) the optimization procedure and (iv) the model for processing by interpolation.

Amongst the various algorithms developed for image registration so far, techniques based on image intensity values are particularly outstanding as they are easy to mechanize as solutions to optimization problems. Pure translations, for example, can be estimated proficiently and universally as the maxima of the cross correlation function between two images [12] [16] [18]. Additional commands such as rotations, combined with scaling, shearing, give rise to nonlinear functions which must be determined using iterative nonlinear optimization methods [12].

In the Medical Imaging Field, image registration is normally used to merge the complementary and synergistic information of images accomplished from multi modalities. A problem when registering image data is that one does not have direct access to the density functions of the image intensities. They must be predictable from the image data. A variety of image registration procedures have been exercised for successfully registering images that are unoccluded and commonly performed with the use of Parzen Windows or Normalized Frequency Histograms [13].

The work proposed in this paper employs Fast Walsh Hadamard Transform (FWHT) [19, 20] for image registration. The coefficients acquired are normalized to find out a unique number which in turn stands for the numerals in a particular range. The tests conducted on clinical images show that proposed algorithm performed well than the conventional Walsh Transform (WT) method in Medical Image Registration. In addition, this paper gives a comparative analysis of FWHT and WT in Medical Image Registration.

The rest of the paper is organized as follows. Section 2 gives an overview on the related work for image registration. Section 3 gives details about WT in image registration. Section 4 describes the proposed approach for image registration using FWHT. Section 5 illustrates the experimental results to prove the efficiency of the proposed approach in image registration and Section 6 concludes the paper with a discussion. 


\section{RELATED WORK}

Many discussions have been carried out previously on Image Registration. This section of paper provides an immediate look on the significant research work in image registration.

An automatic scheme using global optimization technique for retinal image registration was put forth by Matsopoulos et al. in [1]. A robust approach that estimates the affine transformation parameters necessary to register any two digital images misaligned due to rotation, scale, shear, and translation was proposed by Wolberg and Zokai in [2]. Zhu described an approach by cross-entropy optimization in [3]. Jan Kybic and Michael Unser together put forth an approach for fast elastic multidimensional intensity-based image registration with a parametric model of the deformation in [4]. Bentoutou et al. in [5] offered an automatic image registration for applications in Remote Sensing. A novel approach that addresses the range image registration problem for views having low overlap and which may include substantial noise for image registration was described by Silva et al. in [6]. Matungka et al. proposed an approach that involved Adaptive Polar Transform (APT) for Image registration in $[7,10]$. A feature-based fully non supervised methodology dedicated to the fast registration of medical images was described by Khaissidi et al. in [8]. Wei Pan et al. in [9] proposed a technique for image registration using Fractional Fourier Transform (FFT). D.Sasikala and R.Neelaveni in [10] proposed a technique for image registration using Fast Walsh Hadamard Transform (FWHT).

\section{WALSh TRANSFORM}

Orthogonal transforms develop an image into sets of orthogonal basis images each of which represents a type of local structure. Examples are the Walsh, Haar [14], etc. The coefficients of such an extension point towards the effectiveness of the occurrence of the similar structure at the particular position. If these coefficients are normalized by the dc coefficient of the expansion, i.e., the local average gray value of the image, then they measure purely the local structure independent of modality. Walsh basis functions correspond to local structure, in the form of positive or negative going horizontal or vertical edge, corner of a certain type, etc. Registration schemes based on wavelet coefficient matching do not present a general mechanism of combining the matching results across different scales.

Two images $I_{1}$ and $I_{2}, I_{1}$ is assumed as reference image whereas $I_{2}$ represent an image that has to be deformed to match $\mathrm{I}_{1}$. First, consider each pixel, excluding border pixels, a $3 X 3$ neighborhood and compute from it, the nine Walsh coefficients ( $3 X 3$ WT of a $3 X 3$ image patch). If ' $f$ ' is the input image, the matrix of coefficients ' $g$ ' computed for it using equation (1),

$$
g=\left(W^{-1}\right)^{T} \cdot f W^{-1}
$$

Matrix encloses the coefficients of the expansion of the image, in terms of the basis images as in Figure.1 (a) formed by taking the vector outer products of the rows of matrix W[14]. Coefficients are denoted by $\mathrm{a}_{00}, \mathrm{a}_{01}, \mathrm{a}_{02}, \mathrm{a}_{10}, \mathrm{a}_{11}, \mathrm{a}_{12}, \mathrm{a}_{20}, \mathrm{a}_{21}, \mathrm{a}_{22}$, in a matrix form as in Figure. $1(b)$ and take the value in the range $[0,9] . \alpha_{\mathrm{ij}}$ is normalization given in equation (2) makes the method robust to global levels of change of illumination. $a_{00}$ coefficient is the local average gray value of the image, $a_{i j}$ builds coefficients that depicts the local structure.

$$
\alpha \mathrm{ij}=\mathrm{aij} / \mathrm{a} 00
$$

However, the information having intense features and rigid body transformation allows for plenty of redundancy in the system and formulates it robust to noise and bad matches of individual pixels which effectively represent short of local information. Build a distinctive number out of eight numbers using these numbers as the digits of the unique number. The number of levels depends on the number system implemented. For decimal system, the normalized coefficients are quantized that obtain integer values in the range $[0,9]$.

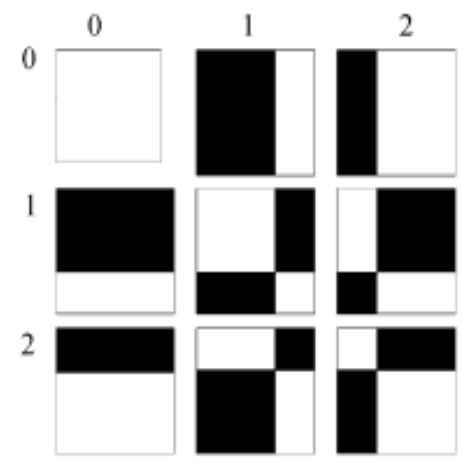

(a). WTs basis images for a $3 \mathrm{X} 3$ images

\begin{tabular}{|l|l|l|}
\hline $\mathbf{a}_{00}$ & $\mathbf{a}_{01}$ & $\mathbf{a}_{02}$ \\
\hline $\mathbf{a}_{10}$ & $\mathbf{a}_{11}$ & $\mathbf{a}_{12}$ \\
\hline $\mathbf{a}_{20}$ & $\mathbf{a}_{21}$ & $\mathbf{a}_{22}$ \\
\hline
\end{tabular}

(b). Nine coefficients in matrix form Figure 1. Walsh Transformation

In Figure 1(a) the coefficients along the first row and the first column are of equal importance, as they assess the existence of a vertical or a horizontal edge, respectively. The remaining four coefficients determine the existence of a corner. The following ordering of coefficients are used in images,

Ordering IA $\alpha_{01}, \alpha_{10}, \alpha_{20}, \alpha_{02}, \alpha_{11}, \alpha_{21}, \alpha_{12}, \alpha_{22}$

Ordering IB $\alpha_{10}, \alpha_{01}, \alpha_{02}, \alpha_{20}, \alpha_{11}, \alpha_{12}, \alpha_{21}, \alpha_{22}$

Ordering IIA $\alpha_{22}, \alpha_{21}, \alpha_{12}, \alpha_{11}, \alpha_{02}, \alpha_{20}, \alpha_{10}, \alpha_{01}$

Ordering IIB $\alpha_{22}, \alpha_{12}, \alpha_{21}, \alpha_{11}, \alpha_{20}, \alpha_{02}, \alpha_{01}, \alpha_{10}$

\section{Proposed APPROACH}

\section{Fast Walsh Hadamard Transform}

A fast transform algorithm is spot as a sparse factorization of the transform matrix, and refers to each factor as a stage. The proposed algorithms have a usual interconnection pattern between stages, and subsequently, the inputs and outputs for each stage are dealt with from (or) to the same locations and the factors of the disintegration, the stages, have the property of being equal between them. The $2 \mathrm{X} 2$ Hadamard matrix is defined as $\mathrm{H}_{2}$ is given in equation (3)

$$
H_{2}=\left[\begin{array}{cc}
1 & 1 \\
1 & -1
\end{array}\right]
$$

A set of radix-R factorizations in terms of identical sparse matrices rapidly obtained from the WHT property that relates the matrix $\mathrm{H}$ with its inverse and is given in equation (4),

$$
H R^{n}=R^{n}\left(H R^{n}\right)^{-1}
$$

$$
\begin{aligned}
& \text { Where } \mathrm{HR}^{\mathrm{n}}=\text { radix- } \mathrm{R} \text { Walsh Hadamard transform; } \\
& \qquad \mathrm{R}^{\mathrm{n}}=\text { radix- } \mathrm{R} \text { factorizations; } \\
& \mathrm{n}=\text { input element; }
\end{aligned}
$$

The FWHT is used to obtain the local structure of the images. This basis function can be helpfully used to get the digital numbers in 
the sense of coefficients [19] [20]. If these coefficients are normalized by the dc coefficient of the expansion, i.e., the local average gray value of the image, then they measure purely the local structure independent of modality. These digits are then normalized to obtain the unique number that is used as feature for image registration. The accomplishment of FWHT voluntarily reduces the time consumption for medical image registration when comparing the same with conventional WT technique for image registration.

\section{EXPERIMENTAL RESULT}

A series of experiments is executed using medical images. The tests are performed using different images of different sizes. A set of $\mathrm{CT}$ and magnetic resonance (MR) medical images that represent the head of the same patient is considered. The original size of these images is given as pixels. In order to eliminate the background parts and the head outline, the original images are cropped, creating subimages of different dimension pixels.

In probability theory and information theory, Mutual Information (sometimes known as transinformation) between two discrete random variables is termed as the amount of information shared between the two random variables. It is a dimensionless quantity with units of bits and can be the reduction in uncertainty. High MI indicates a large reduction in uncertainty; low MI indicates a small reduction; and zero MI between two random variables means the variables are independent.

$$
\begin{gathered}
\text { Mutual Information } \\
I(X ; Y)=\sum_{y \in Y} \sum_{x \in X} p(x, y) \log \left(\frac{p(x, y)}{p_{1}(x) p_{2}(y)}\right)
\end{gathered}
$$

$X$ and $Y$ - Two discrete random variables.

$p(x, y)$ - Joint probability distribution function of $X$ and $Y$.

$p 1(x)$ and $p 2(y)$ - Marginal probability distribution functions of $X$ and $Y$ respectively.

The Correlation Coefficient is from statistics, is a measure of how well the predicted values from a forecast model "fit" with the real-life-data. If there is no relationship between the predicted values and actual values the $\mathrm{CC}$ is very low. As the strength of the relationship between the predicted values and actual values increases, so does the CC. Thus higher the $\mathrm{CC}$ the better it is.

\section{Correlation Coefficient}

$C(t, s ; \theta)=\frac{\sum_{x} \sum_{y} I_{1}^{n e w}(x, y)-\overline{I_{1}^{n e w}(x, y)} I_{2}^{n e w}(x \cos \theta-y \sin \theta-t, x \sin \theta+y \cos \theta-s)-\overline{\left.I_{2}^{n e w}(x, y)\right]}}{\sqrt{\sum_{x} \sum_{y} I_{1}^{n e w}(x, y)-{\overline{\left.I_{1}^{n e w}(x, y)\right]}}^{2} \sum_{x} \sum_{y} I_{2}^{n e w}(x \cos \theta-y \sin \theta-t, x \sin \theta+y \cos \theta-s)-{\overline{I_{2}^{n e w}}(x, y)}^{2}}}$

$$
\begin{aligned}
& I_{1}^{\text {new }}, I_{2}^{\text {new }}-\quad \text { Two new images that differ from each other by rotation } \\
& \mathrm{t}, \mathrm{s} \quad \text { - } \quad \text { Shifting parameters between the two images. } \\
& \theta \quad \text { - Rotation angle. } \\
& \text { new }(x, y) \text { - Average structure value of the pixels in the } \\
& I_{1}^{\text {new }}(x, y), I_{2}^{\text {new }}(x, y) \text { overlapping parts of images } \\
& \text { respectively. } \\
& I_{1}^{\text {new }}(x, y), I_{2}^{\text {new }}(x, y)
\end{aligned}
$$

Each type of image is taken as reference image i.e., using CT Axial and CT Sagittal, MRI-T1 Axial, MRI-T1 Sagittal and MRI-T1 Frontal and MRI-T2 Axial, MRI-T2 Sagittal and MRI-T2 Frontal images are considered and each image is translated by $(-5,5)$ coordinates and also each image is rotated by 5 degrees in angle to get the transformed image. Using reference image and its corresponding transformed image the registered image is obtained after registration. The performance indexes MI, CC and the Elapsed Time taken for registration are obtained for both WT and FWHT. The analysis is done using these performance indexes.

During image registration, Figures 2(a) to 9(a) shows the registered images and same while registering using both WT \& FWHT. Also Figures 2.(b) to 9(b) shows difference in images and it is also same while registering using both WT \& FWHT.

i) CT - Registered Axial image - $500 \times 653$ - 77k -JPEG.
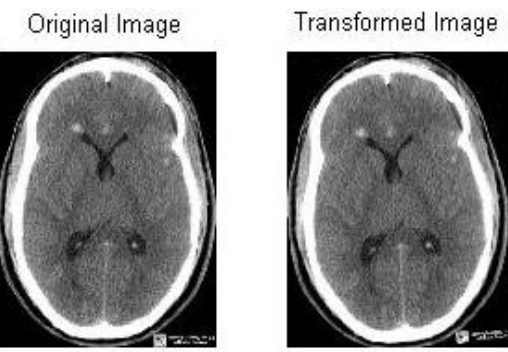

Registered Image

a) Registered Image obtained for using WT \& FWHT

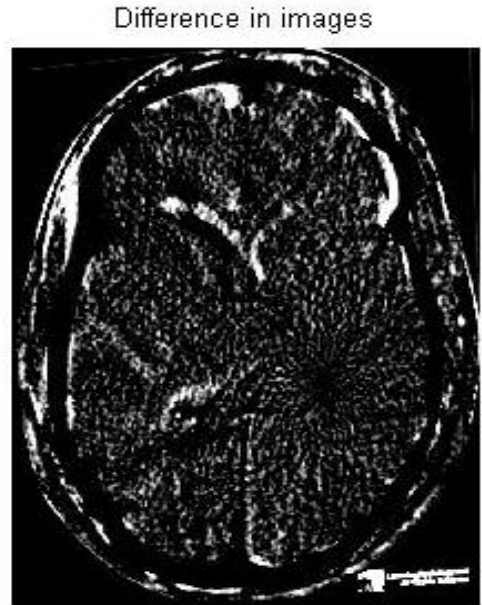

b) Difference in images obtained for using WT \& FWHT Figure 2. Images obtained using WT \& FWHT

(ii) CT - Registered Sagittal image - 840 x 754 - 69k - JPEG


Registered Image

a) Registered Image obtained using WT \& FWHT

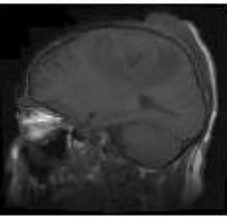




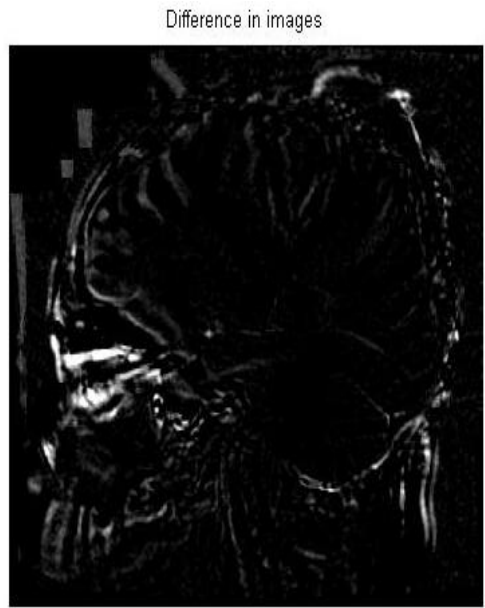

b)Difference in images obtained using WT\& FWHT

Figure 3. Images obtained using WT\& FWHT

(iii) MRI T1-Registered Axial image - 459 x 504 - 62k - JPEG
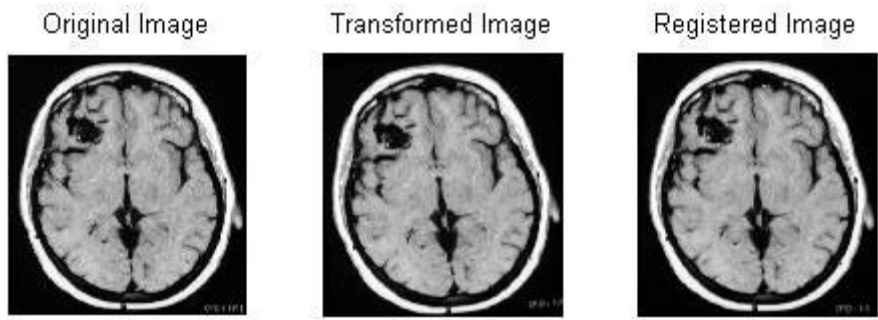

a) Registered Image obtained using WT \& FWHT

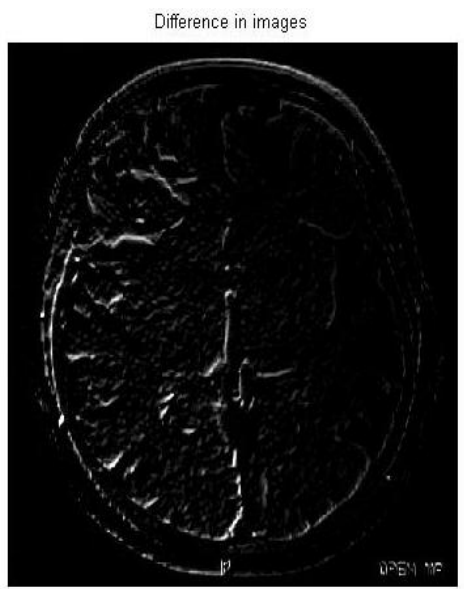

b)Difference in images obtained using WT \& FWHT Figure 4. Images obtained using WT \& FWHT

(iv) MRI T1-Registered Sagittal image - 350 x 350 - 28k - JPEG
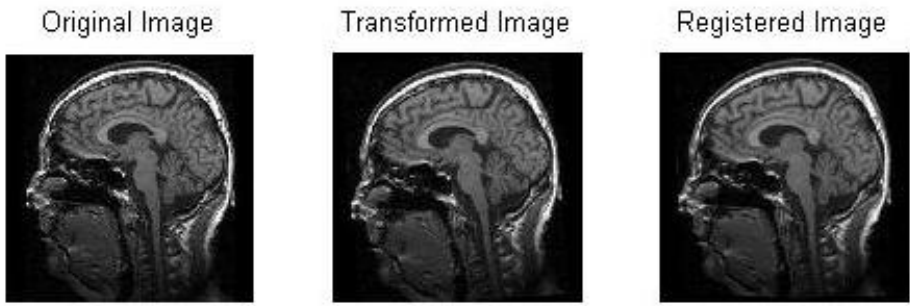

a) Registered Image obtained using WT \& FWHT

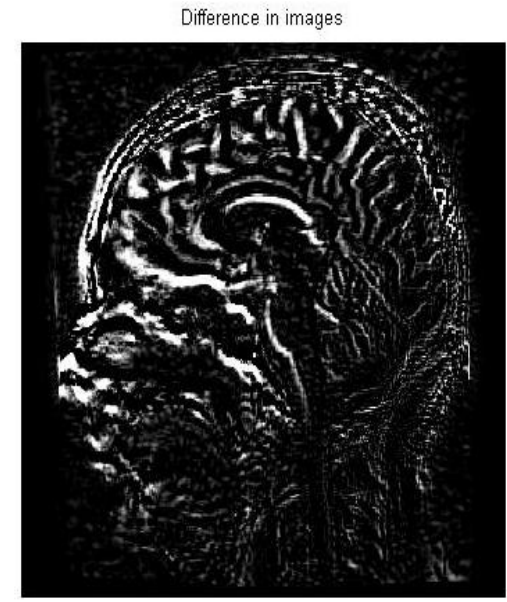

b)Difference in images obtained using WT \& FWHT Figure 5. Images obtained using WT \& FWHT

(v) MRI T1-Registered Frontal image - 400 x 400 - 18k - JPEG
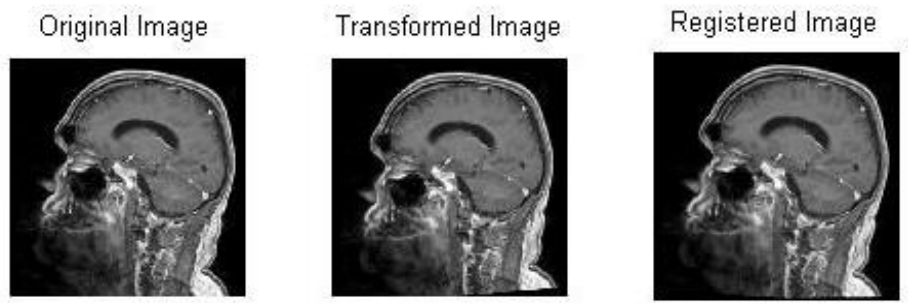

b) Registered Image obtained using WT \& FWHT

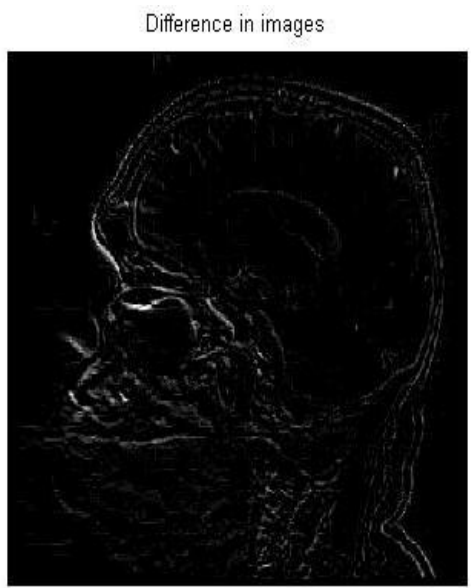

b)Difference in images obtained using WT \& FWHT Figure 6. Images obtained using WT \& FWHT

(vi) MRI T2-Registered Axial image - 350 x 350 - 24k - JPEG

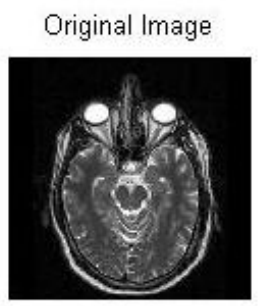

Transformed Image

Registered Image

a) Registered Image obtained using WT \& FWHT
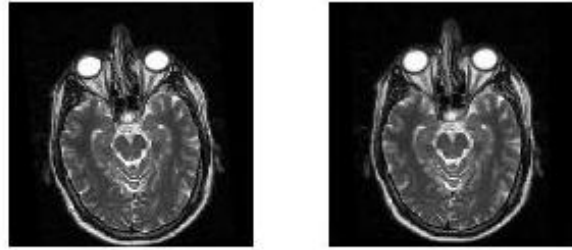
Difference in images

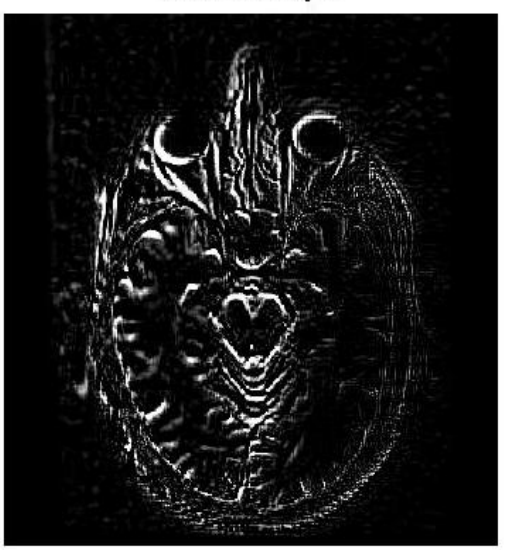

b) Difference in images obtained using WT \& FWHT

Figure7. Images obtained using WT \& FWHT

(vii) MRI T2-Registered Axial image - 831 x 969 - 328k - JPEG


a) Registered Image obtained using WT \& FWHT Difference in images

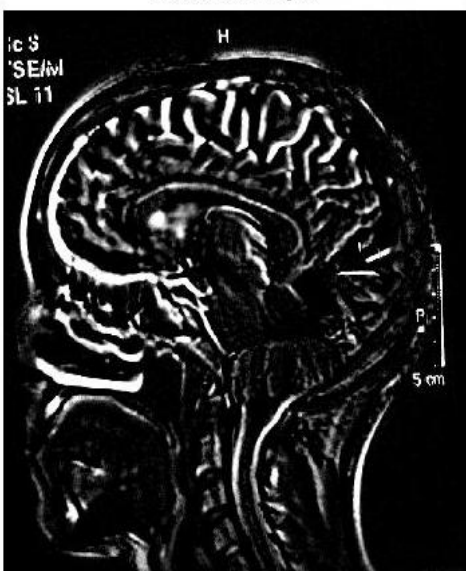

b)Difference in images obtained using WT \& FWHT

Figure8. Images obtained using WT \& FWHT

(viii) MRI T2-Registered Frontal image - 390 x 448- 37k - JPEG
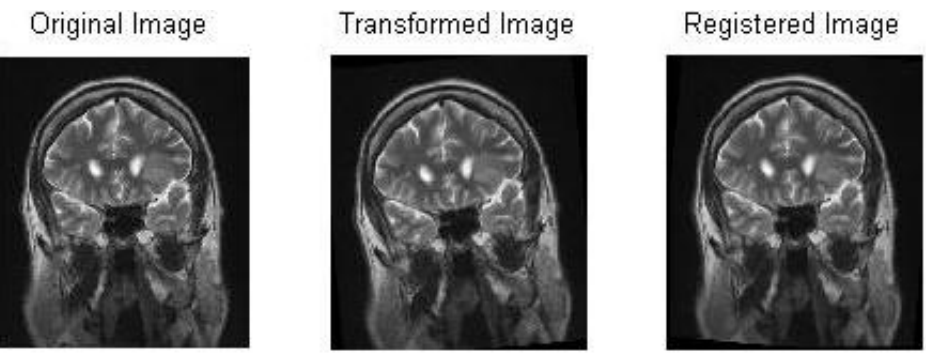

a)Registered Image obtained using WT \& FWHT

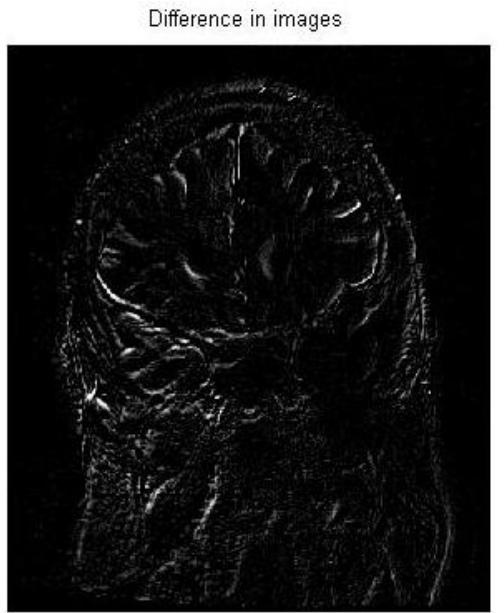

b)Difference in images obtained using WT \& FWHT Figure9. Images obtained using WT \& FWHT

Table 1. Mutual Information of WT and FWHT.

\begin{tabular}{|c|c|c|}
\hline Images & $\begin{array}{l}\text { MI after } \\
\text { registration } \\
\text { for Walsh } \\
\text { Transform }\end{array}$ & $\begin{array}{l}\text { MI after } \\
\text { registration } \\
\text { for Fast } \\
\text { Walsh } \\
\text { Hadamard } \\
\text { Transform }\end{array}$ \\
\hline $\begin{array}{l}\text { i) CT - Registered Axial image }-500 \\
\text { x } 653-77 \mathrm{k}-\text { JPEG. }\end{array}$ & 1.4402 & 1.4657 \\
\hline $\begin{array}{l}\text { (ii) CT - Registered Sagittal image - } 840 \\
\text { x } 754-69 \mathrm{k}-\text { JPEG }\end{array}$ & 1.8720 & 1.9640 \\
\hline $\begin{array}{l}\text { (iii) MRI T1-Registered Axial image - } \\
459 \text { x } 504-62 \mathrm{k} \text { - JPEG }\end{array}$ & 3.0650 & 4.4288 \\
\hline $\begin{array}{l}\text { (iv) MRI T1-Registered Sagittal image - } \\
350 \times 350-28 \mathrm{k}-\text { JPEG }\end{array}$ & 1.5071 & 1.3349 \\
\hline $\begin{array}{l}\text { (v) MRI T1-Registered Frontal image - } \\
400 \text { x } 400 \text { - 18k - JPEG }\end{array}$ & 4.1333 & 3.1546 \\
\hline $\begin{array}{l}\text { (vi) MRI T2-Registered Axial image - } \\
350 \times 350-24 \mathrm{k}-\text { JPEG }\end{array}$ & 2.0027 & 1.9237 \\
\hline $\begin{array}{l}\text { (vii) MRI T2-Registered Axial image - } \\
831 \text { x } 969-328 \mathrm{k}-\text { JPEG }\end{array}$ & 1.0677 & 0.8253 \\
\hline $\begin{array}{l}\text { (viii) MRI T2-Registered Frontal image } \\
-390 \times 448-37 \mathrm{k}-\text { JPEG }\end{array}$ & 3.4249 & 2.4449 \\
\hline
\end{tabular}

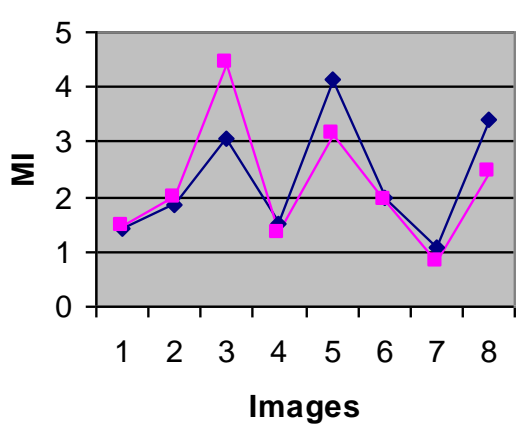

Figure 10.Comparison of WT and FWHT using MI. 
Table 2. Correlation Coefficient of WT and FWHT.

\begin{tabular}{|l|c|c|}
\hline \multicolumn{1}{|c|}{ Images } & $\begin{array}{c}\text { CC after } \\
\text { registration } \\
\text { for Walsh } \\
\text { Transform }\end{array}$ & $\begin{array}{c}\text { CC after } \\
\text { registration } \\
\text { for Fast } \\
\text { Walsh } \\
\text { Hadamard } \\
\text { Transform }\end{array}$ \\
\hline $\begin{array}{l}\text { i) CT - Registered Axial image - 500 x } \\
\text { 653 - 77k -JPEG. }\end{array}$ & 0.9093 & 0.9138 \\
\hline $\begin{array}{l}\text { (ii) CT - Registered Sagittal image - 840 x } \\
\text { 754 - 69k - JPEG }\end{array}$ & 0.9285 & 0.9388 \\
\hline $\begin{array}{l}\text { (iii) MRI T1-Registered Axial image - 459 } \\
\text { x 504 - 62k - JPEG }\end{array}$ & 0.9939 & 0.9993 \\
\hline $\begin{array}{l}\text { (iv) MRI T1-Registered Sagittal image - } \\
\text { 350 x 350 - 28k - JPEG }\end{array}$ & 0.8572 & 0.8271 \\
\hline $\begin{array}{l}\text { (v) MRI T1-Registered Frontal image - } \\
\text { 400 x 400 - 18k - JPEG }\end{array}$ & 0.9843 & 0.9804 \\
\hline $\begin{array}{c}\text { (vi) MRI T2-Registered Axial image - 350 } \\
\text { X 350 - 24k - JPEG }\end{array}$ & 0.9262 & 0.9181 \\
\hline $\begin{array}{c}\text { (vii) MRI T2-Registered Axial image - } \\
\text { 831 x 969 - 328k - JPEG }\end{array}$ & 0.7917 & 0.7213 \\
\hline $\begin{array}{c}\text { (viii) MRI T2-Registered Frontal image - } \\
\text { 390 448- 37k - JPEG }\end{array}$ & 0.9864 & 0.9718 \\
\hline
\end{tabular}

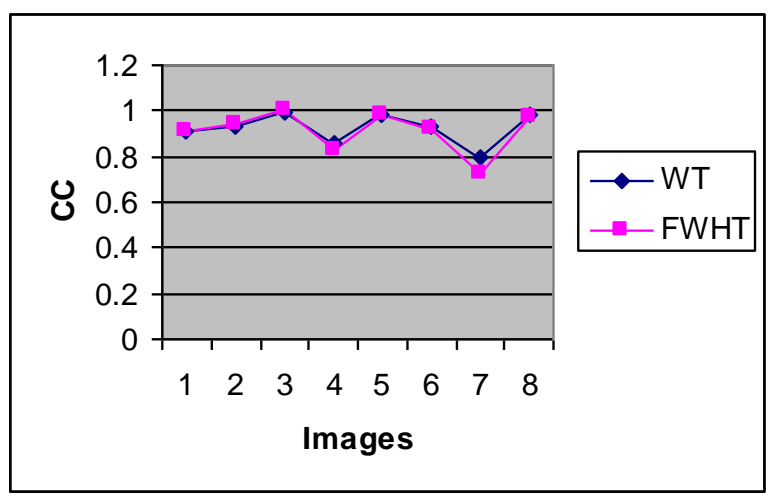

Figure11.Comparison of WT and FWHT using CC.

Table 3. Time Comparison of WT and FWHT.

\begin{tabular}{|c|c|c|}
\hline \multicolumn{1}{|c|}{ Images } & $\begin{array}{c}\text { Elapsed } \\
\text { Time in Secs } \\
\text { after } \\
\text { registration } \\
\text { for Walsh } \\
\text { Transform }\end{array}$ & $\begin{array}{c}\text { Elapsed } \\
\text { Time in Secs } \\
\text { after } \\
\text { registration } \\
\text { for Fast } \\
\text { Walsh } \\
\text { Hadamard } \\
\text { Transform }\end{array}$ \\
\hline $\begin{array}{c}\text { i) CT - Registered Axial image - } \\
\text { 500 x 653 - 77k -JPEG. }\end{array}$ & 238.312 & 7.328 \\
\hline $\begin{array}{l}\text { (ii) CT - Registered Sagittal image - } \\
\text { 840 x 754 - 69k - JPEG }\end{array}$ & 522.625 & 13.562 \\
\hline $\begin{array}{c}\text { (iii) MRI T1-Registered Axial image } \\
\text {-459 x 504 - 62k - JPEG }\end{array}$ & 163.61 & 5.641 \\
\hline $\begin{array}{l}\text { (iv) MRI T1-Registered Sagittal } \\
\text { image - 350 x 350 - 28k - JPEG }\end{array}$ & 86.281 & 2.828 \\
\hline $\begin{array}{c}\text { (v) MRI T1-Registered Frontal } \\
\text { image - 400 x 400 - 18k - JPEG }\end{array}$ & 110.391 & 3.75 \\
\hline $\begin{array}{c}\text { (vi) MRI T2-Registered Axial image } \\
\text { - 350 x 350 - 24k - JPEG }\end{array}$ & 83.047 & 3.109 \\
\hline $\begin{array}{c}\text { (vii) MRI T2-Registered Axial } \\
\text { image - 831 x 969 - 328k - JPEG }\end{array}$ & 754.203 & 17.797 \\
\hline $\begin{array}{c}\text { (viii) MRI T2-Registered Frontal } \\
\text { image - 390 x 448- 37k - JPEG }\end{array}$ & 123.61 & 4.328 \\
\hline
\end{tabular}

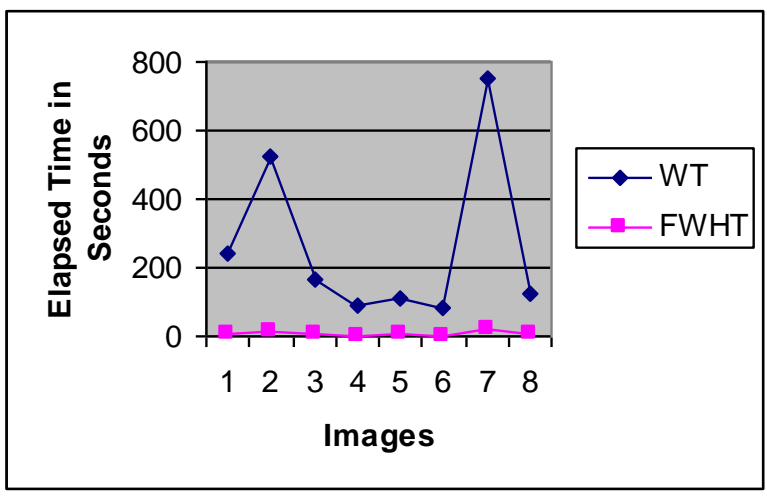

Figure 12.Comparison of WT and FWHT in terms of time

The quality of registration is based on the value of MI and CC which is mentioned before. The high values of MI and CC represents the better quality of registration. When different Images are used and tested with various values of translated, and / or rotated images, it proves that FWHT registration of brain images is better than WT registration for brain images. Hence when considering the overall performance of geometrical forward transformations it shows that FWHT registration is better when compared to WT registration. This is proved using the recorded values available in the tables and also from the graphical figures drawn using these recorded values.

Table 1 show the summary of all the results when a single ordering is taken into account using WT and FWHT in terms of MI. MI represents Mutual Information [16]. CC represents Correlation Coefficient. Figure 10.shows the performance comparison of WT and FWHT with respect to MI. Table 2 represents the summary of all results using conventional WT and FWHT in terms of CC. Table 3 indicates the Elapsed time consumption for registering image using conventional WT and FWHT. Figure 11. represents the comparison of conventional WT and FWHT in terms of CC. Figure 12 represents the Elapsed time consumption for registering image using conventional WT and FWHT.

From the above analysis it proves that the performance of the FWHT is better than the WT in terms the performance indexes, CC and Elapsed Time of registration obtained from the experimental results. Here MI for CT images is better for FWHT than WT, but for MRI-T1 and MRI-T2 images WT is better than FWHT. From the analysis it proves that FWHT is the better than WT in terms of Elapsed Time Taken for registration.

\section{Conclusion}

This work suggests a new algorithm for medical image registration. A Fast Walsh Hadamard Transform is proposed in this paper for medical image registration. This transform decreases the time consumption and increases $\mathrm{MI}$ and $\mathrm{CC}$ in image registration. Therefore it confirms to be a better approach for medical image registration than any other traditional Walsh Transform. The coefficients acquired using this transform are then normalized to obtain the unique digit. This unique digit characterizes the local structure of an image. Moreover this unique digit shows the feature of an image for image registration. The experimental results revealed the fact that the proposed algorithm using Fast Walsh Hadamard Transform executed well in image registration. The future work concentrates on additional improvement in the results by using some other transforms that utilize correlation coefficients. 


\section{REFERENCES}

[1] George K. Matsopoulos, Nicolaos A. Mouravliansky, Konstantinos K. Delibasis, and Konstantina S. Nikita, "Automatic Retinal Image Registration Scheme Using Global Optimization Techniques," IEEE Transactions on Information Technology in Biomedicine, vol. 3, no. 1, pp. 47-60, 1999.

[2] G. Wolberg, and S. Zokai, "Robust image registration using logpolar transform," Proceedings of International Conference on Image Processing, vol. 1, pp. 493-496, 2000.

[3] Yang-Ming Zhu, "Volume Image Registration by Cross-Entropy Optimization," IEEE Transactions on Medical Imaging, vol. 21, no. 2, pp. 174-180, 2002.

[4] Jan Kybic, and Michael Unser, "Fast Parametric Elastic Image Registration," IEEE Transactions on Image Processing, vol. 12, no. 11, pp. 1427-1442, 2003.

[5] Y. Bentoutou, N. Taleb, K. Kpalma, and J. Ronsin, "An Automatic Image Registration for Applications in Remote Sensing," IEEE Transactions on Geosciences and Remote Sensing, vol. 43, no. 9, pp. 2127-2137, 2005.

[6] Luciano Silva, Olga R. P. Bellon, and Kim L. Boyer, "Precision Range Image Registration Using a Robust Surface Interpenetration Measure and Enhanced Genetic Algorithms," IEEE Transactions on Pattern Analysis and Machine Intelligence, vol. 27, no. 5, pp. 762-776, 2005.

[7] R. Matungka, Y. F. Zheng, and R. L. Ewing, "Image registration using Adaptive Polar Transform," 15th IEEE International Conference on Image Processing, ICIP 2008, pp. 2416-2419, 2008.

[8] G. Khaissidi, H. Tairi and A. Aarab, "A fast medical image registration using feature points," ICGST-GVIP Journal, vol. 9, no. 3, 2009.

[9] Wei Pan, Kaihuai Qin, and Yao Chen, "An Adaptable-Multilayer Fractional Fourier Transform Approach for Image Registration," IEEE Transactions on Pattern Analysis and Machine Intelligence, vol. 31, no. 3, pp. 400-413, 2009.

[10] D.Sasikala and R.Neelaveni, "Registration of Brain Images using Fast Walsh Hadamard Transform", International Journal of Computer Science and Information Security, Vol. 8, No. 2, May 2010, pp 96-105.

[11] R. Matungka, Y. F. Zheng, and R. L. Ewing, "Image registration using Adaptive Polar Transform," IEEE Transactions on Image Processing, vol. 18, no. 10, pp. 2340-2354, 2009.

[12] Jr. Dennis M. Healy, and Gustavo K. Rohde, "Fast Global Image Registration using Random Projections," 4th IEEE International Symposium on Biomedical Imaging: From Nano to Macro, ISBI 2007, pp. 476-479, 2007.

[13] C. Fookes and A. Maeder, "Quadrature-Based Image Registration Method using Mutual Information," IEEE International Symposium on Biomedical Imaging: Nano to Macro, vol. 1, pp. 728-731, 2004.
[14] M. Petrou and P. Bosdogianni, Image Processing-The Fundamentals. New York: Wiley, 1999.

[15] Pere Marti-Puig, “A Family of Fast Walsh Hadamard Algorithms With Identical Sparse Matrix Factorization," IEEE Transactions on Signal Processing Letters, vol. 13, no. 11, pp. 672-675, 2006.

[16] J. L. Moigne, W. J. Campbell, and R. F. Cromp, “An automated parallel image registration technique based on correlation of wavelet features," IEEE Trans. Geosci. Remote Sens., vol. 40, no. 8, pp. 1849-1864, Aug. 2002.

[17] J. P. W. Pluim, J. A. Maintz, and M. A. Viergever, "Image registration by maximization of combined mutual information and gradient information," IEEE Trans. Med. Imag., vol. 19, no. 8, pp. 899-814, Aug. 2000.

[18] Z. Zhang, J. Zhang, M. Liao, and L. Zhang, "Automatic registration of multi-source imagery based on global image matching," Photogramm. Eng. Remote Sens., vol. 66, no. 5, pp. 625-629, May 2000.

[19] M. Bossert, E. M. Gabidulin, and P. Lusina, "Space-time codes based on Hadamard matrices proceedings," in Proc. IEEE Int. Symp. Information Theory, Jun. 25-30, 2000, p. 283.

[20] L. Ping, W. K. Leung, and K. Y. Wu, "Low-rate turbo-Hadamard codes," IEEE Trans. Inf. Theory, vol. 49, no. 12, pp. 3213-3224, Dec. 2003.

\section{AUTHORS PROFILE}

D.Sasikala is presently working as Assistant Professor, Department of CSE, Bannari Amman Institute of Technology, Sathyamangalam. She received B.E.( CSE) from Coimbatore Institute of Technology, Coimbatore and M.E. (CSE) from Manonmaniam Sundaranar University, Tirunelveli. She is now pursuing Phd in Image Processing. She has 11.5 years of teaching experience and has guided several UG and PG projects. She is a life member of ISTE. Her areas of interests are Image Processing, System Software, Artificial Intelligence, and Compiler Design.

R. Neelaveni is presently working as a Associate Professor, Department of EEE, PSG College of Technology, Coimbatore. She has a Bachelor's degree in ECE, a Master's degree in Applied Electronics and $\mathrm{PhD}$ in Biomedical Instrumentation. She has 23 years of teaching experience and has guided many UG and PG projects. Her research and teaching interests includes Applied Electronics, Analog VLSI, Computer Networks, and Biomedical Engineering. She is a Life member of Indian Society for Technical Education (ISTE). She has published several research papers in International, National Journals and Conferences. 